\title{
The Past, Present and Future of Mathematics in China and India
}

\author{
by Shing-Tung Yau
}

Above is the title of the talk that Professor Ravindra Kulkarni asked me to give. It is a tall order as I am not a professionally trained historian, although I like to read history in my leisure. Naturally, I am more familiar with the mathematical achievements of China than of other countries. In reading through some of the writings by Western historians on mathematics, I am often surprised by the somewhat biased discussions towards the mathematical achievements of Asia.

There is no question that the ancient Greeks made numerous fundamental contributions to mathematics, as we can see many of them in use in our present day. However, many of the Greek manuscripts were partially destroyed during the early centuries, and therefore, it takes great efforts to identify the form of the original manuscripts. The Romans, the Byzantines, the Arabs and the Moors all spent much efforts to translate and circulate the classic Greek manuscripts.

Often, great ancient books incorporate a collection of many people's works and were sometimes edited by a number of authors. Commenting on the Euclid's Elements (believed to be written around $300 \mathrm{BC}$ ), the Greek mathematician Proclus of fifth century AD wrote: "Euclid, who put together the Elements, collecting many of Eudoxus' theorems, perfecting many of Theaetetus', and also bringing to irrefragable demonstration the things which were only somewhat loosely proved by his predecessors." There are copies of Euclid's Elements in Greek that are in the Vatican Library of the Holy See and the Bodleian Library of Oxford University. However, they are not complete and of variable quality. (See a fragment of the Elements from around $100 \mathrm{AD}$ in Figure 1. Many hypotheses have been put forth on the contents of the original text based on translations and originals. Similarly, the origins of ancient Chinese books of mathematics such as the Nine Chapters on the Mathematical Art are difficult to know for sure. Most likely, the Nine Chapters is an accumulation of works of many generations and was updated by many people. The recent discovery of an old book dated around 186 BC showed some form of the preliminary version of the Nine Chapters.

There are several outstanding contributions in mathematics that appeared in different cultures. The most notable is the Pythagorean theorem. Pythagoras is believed to be born between 580 to $568 \mathrm{BC}$. This is not much later than the time when the Egyptian papyrus was introduced into Greece around 650 BC. It was said that he traveled to Egypt, Babylon and perhaps even India. Since there are ancient records of the Pythagorean triples in all these countries besides China, it is likely that Pythagoras learned the statement of his theorem from these countries. On the other hand, his great contribution is that he is the first one who proved the theorem. In fact, the concept of a proof based on formal logic found in Greek mathematics is rather unique as compared with other countries. It may be a mystery that countries far apart discovered the same theorem at different levels of depth.

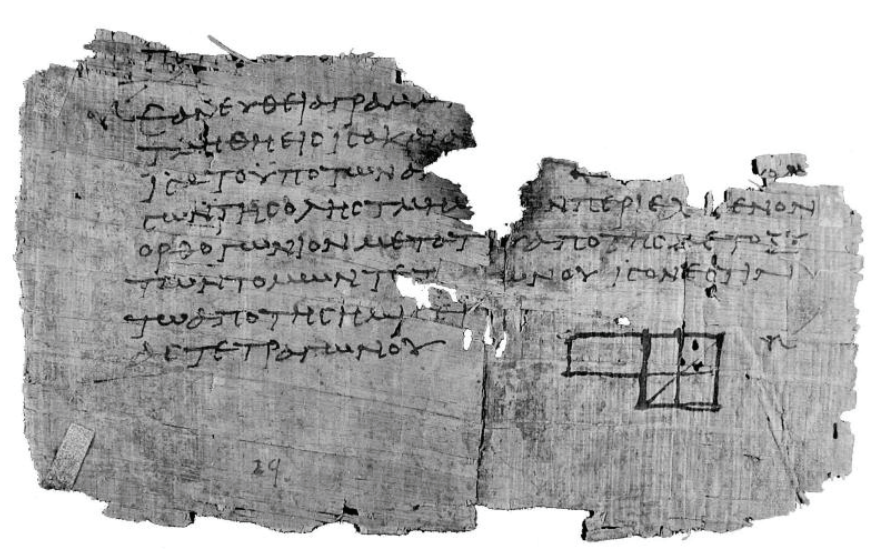

Figure 1. A fragment of papyrus of Euclid's Elements dug up at Oxyrhynchus, Egypt in 1896-1897. It has been dated to between $75 \mathrm{AD}$ and $125 \mathrm{AD}$. (from Bill Casselman's web page)

For the great desire to build a right-angled triangle, it is rather natural to imagine some primitive form of the Pythagorean theorem. Therefore, it is quite possible such a development can be made independent of each other and evolved in its own right.

A great deal of mathematical developments were necessitated by cultural needs, for examples, the calender, astronomy, rituals in the temple, musical instruments, etc. And as a result, mathematical developments can be rather unique to the culture of that country. For example, it is puzzling to me why the ancient Chinese were so much interested in the magic squares. A legend claims that interest in such objects dates back to two thousand BC and continued all the way to the Song Dynasty. On the other hand, the Muslims were very much interested in patterns-tiling of geometric figures-in the years around $1200 \mathrm{AD}$ to $1600 \mathrm{AD}$. It was said that they knew the non-periodic tiling: Penrose tiling. It is quite likely that each culture depending on its needs, developed its 
mathematics and at the same time absorbed ideas from different countries. Moreover, the interactions between countries were likely much more frequent than what we have on records.

Interactions between ancient Egypt, Greece, Babylonia, Persia and India are not that hard to imagine. But the Silk Road between China and the West dates back to the very
It is likely that what the Chinese called the Da Yuezhi in Central Asia was the center of such cultural exchange. The Yuezhi people moved several times. They later conquered what is today Afghanistan and Pakistan in the northwest of India and formed the Kushan Empire from the period of $40 \mathrm{AD}$ to $250 \mathrm{AD}$. For their likely importance in linking the different cultures, we shall spend some time dis-

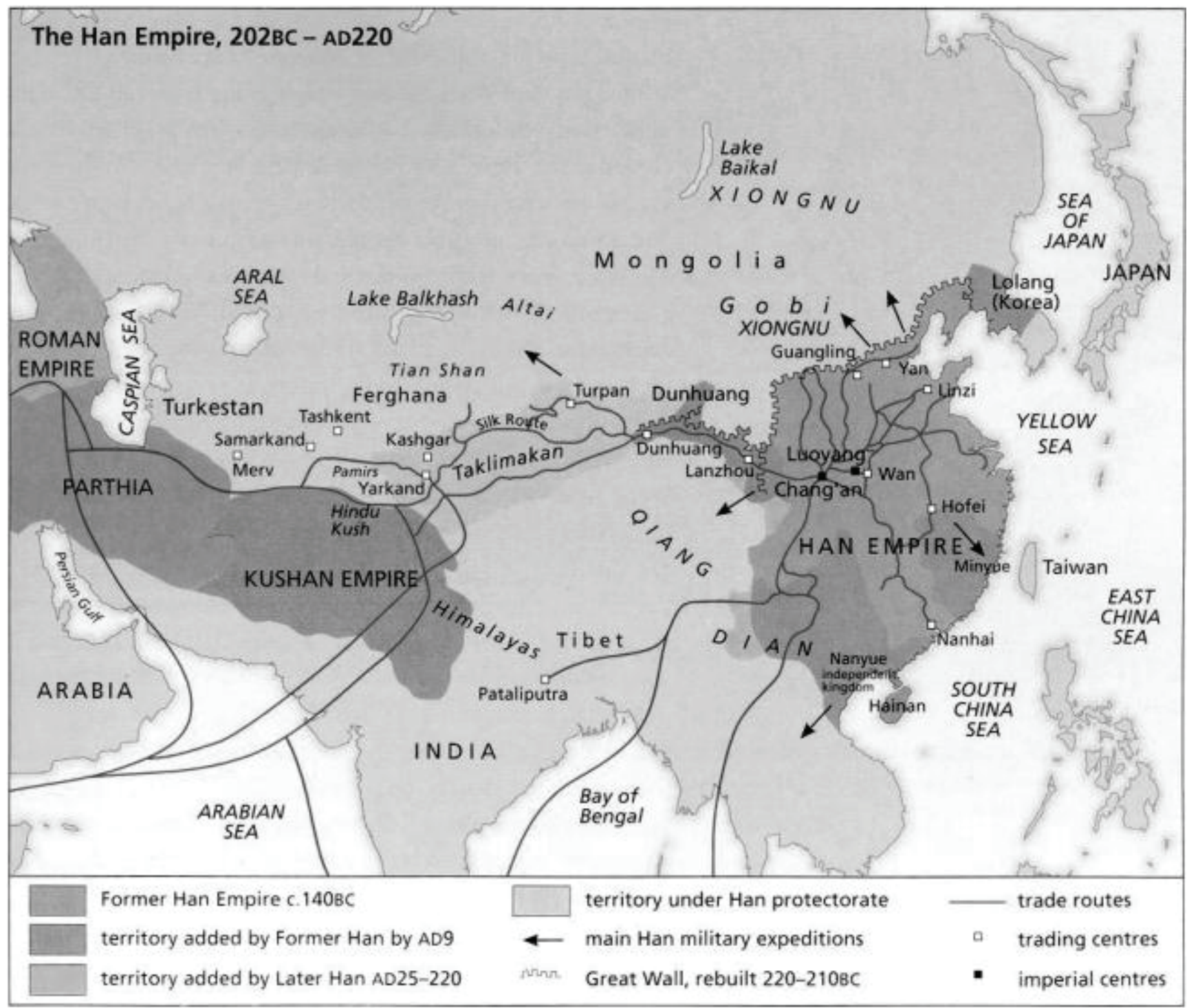

Figure 2. Han and Kushan Empires (from Kenyon College web resources)

early days. For the merchants traveling along the Silk Road, they were usually in groups of one to three hundred people where the engineers were an important core group. The merchants needed the engineers' help to build bridges and overcome any obstacles along the road. These engineers knew mathematics and clearly some communications between engineers from different countries should have occurred.

I would like to propose a hypothesis that the major period of interaction of the ancient cultures with regards to mathematics happened during the period from $300 \mathrm{BC}$ to $200 \mathrm{AD}$ between Persia, Egypt, Greeks, India and China. cussing the Yuezhi people below.

\section{Communications between ancient China and Central Asia}

Let us start by first commenting on the historical documents I can find on the communications between ancient China and Central Asia.

It should be noted that the written documents of ancient China are much less reliable for events that happened before $600 \mathrm{BC}$. It has been speculated that seg- 
ments of some of the ancient books were written during the period of the Han Dynasty (around $200 \mathrm{BC}$ to $200 \mathrm{AD}$ ). A lot of the books were dug up in tombs or walls during the ancient days. But often the original disintegrated and some later authors made up the rest either from memory or by imagination. One has to be very careful how to use such books. There are of course recent discoveries in tombs dating back to two or three hundred $\mathrm{BC}$ that are very reliable. On the other hand, by and large, Chinese government-appointed historians are widely regarded to be very serious in their works. The government supported a large group of historians to collect data. In most cases, the government appointed scholars to study records of previous governments. These are high-level scholars who considered producing honest works to be noble acts in their life. Hence most records given by government historians are rather reliable. There are historical records which were read by Confucius and his disciples. For example, Confucius thought highly of the I-Ching and this book certainly should had existed before $500 \mathrm{BC}$. In fact, recently some ancient fragments of the book I-Ching were
About 1500 BC, the Aryans, who were pastoral people, took over the Harappan culture and developed a language called Sanskrit. This language was systematically organized by Panini around 500 BC. On a basis of 4000 sutras, he built the Sanskrit language. It is interesting to know whether such logical structure has any influence on Euclid's Elements.

Let us now discuss some relevant facts written by Chinese historians.

\section{King Mu, Emperor of Zhou and his western travel}

A classical history book written by Sima Qian around 180 BC is called Shiji or in English Records of the Grand Scribe. Sima Qian was born into a family of professional historians appointed by the emperor. Sima Qian traveled around ancient China to collect and verify historical events. Because of his family's connections, he was able to read many historical documents from centuries past in

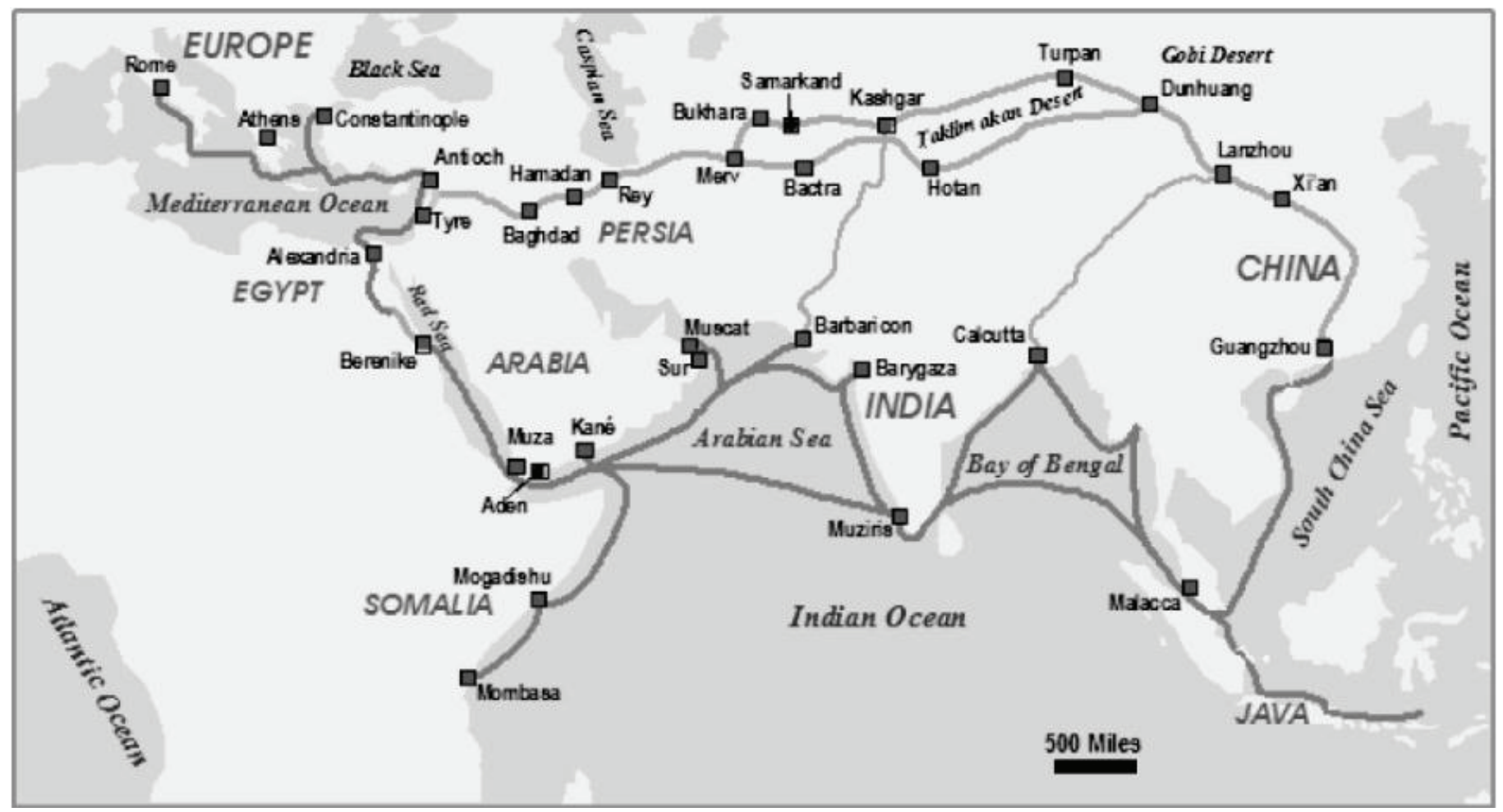

Figure 3. The Silk Road (from the Silk Road Foundation)

dug up on bamboos which can be traced back to 200 BC.

In contrast, Indian literature are more difficult to trace prior to $500 \mathrm{AD}$. For example, it is still not clear what is the exact date of the important Bakhshali manuscript. One may also say that while there is continuity in Chinese history, several parts of Indian subcontinent is less united. Several invasions occurred, especially in northern India. The ancient Harappan script remains today undeciphered. Hence very ancient Indian mathematics proficiency must be based on excavated artifacts. the possession of the government. In his writing about the Kingdom of Zhao, he described the story around 950 BC of the emperor of the Zhou Dynasty, King Mu, who rode fast running horses to western China, all the way to the Qilian Mountains (or in Chinese called Tian Shan, the Heavenly Mountains) to meet Xi Wang $\mathrm{Mu}$, the "Queen Mother of the West," and leader of a local tribe. There are many fairy tales or legends based on this trip. The area that the Zhou emperor went is speculated to be part of Da Yuezhi, a country in Central Asia. The story involves jade 
which is consistent with the traders of Da Yuezhi.

\section{Westerners in ancient China, students of Confucius}

In the book of Shiji, there was an extensive discussion on the students of Confucius. Of the few commentaries on Shiji, an important one was written by Pei Yin called Collected Explanations. This was written around 420 AD. In his commentary, Pei Yin mentioned another commentary which was written by a group of scholars under the leadership of Cao Pei, the King of Wei, around 210 AD. (After a few hundred years, only a small part of this book survived.) In this commentary, it mentioned that when Confucius died, his students traveled from all over the country to stay for three years near his tomb. Each of them brought plants from their home countries to be planted in the tomb garden of Confucius. Some of these plants came from the "western area." This means that students from the West came to study in China. The Han emperors were fond of the people from Central Asia. When the great Han emperor Wudi defeated the Huns (Xiongnu) in a major battle, a king of the Huns was killed by another fellow Huns king. The widow and her son ended up surrendering to the Han Dynasty. The son Jin Richan was eventually promoted to the second most powerful position in the imperial court of the Han around $140 \mathrm{BC}$.

Regarding King $\mathrm{Mu}$ and Westerners in ancient China, the historical evidences for them are not solid and we should be critical about their reliability. However, the historical events that I now describe are backed up with solid evidences.

\section{Han Dynasty, Da Yuezhi, and the Kushan Empire}

The first major communication between China and the Central Asian countries is around the time of Wudi. Wudi was interested in defeating the Huns on the northwest boundary of China. In $138 \mathrm{BC}$, he sent an ambassador named Zhang Qian to a country called Da Yuezhi (moon people) to seek help to defeat the Huns. (The reason for this is that the Yuezhi were defeated by the Huns in a very cruel manner.) These people are believed to be of Indo-European descent and were called Tocharians by ancient Greeks. They eventually migrated to Transoxiana, Bactria and then northwestern India where they formed the Kushan Empire in India (100-300 AD). See Figure 2. It was said in Guanzi, a Chinese book written around 645 BC that they supplied jade to the Chinese from the nearby mountains of Yuezhi at Gansu. (The queens of the Shang Dynasty were fond of jade and the Yuezhi were called the Di people, meaning western barbarians.)

Zhang Qian had about a group of a hundred people that went with him. But he was captured by the Huns and after 10 years of imprisonment, he managed to escape (with one assistant) to see the King of Yuezhi who had no interest to help the Han to defeat the Huns. On the other hand, Zhang Qian spent a year in Yuezhi and wrote down detail descriptions of the area around this country, which is southwest of another country called Dayuan (Ferghana), south of the Gui (Oxus) River. He witnessed the last period of the Greco-Bactrian Kingdom when it was being subjugated by the Yuezhi. He reported about Shendu (or Sindhu in Sanskrit). This was a region of India bordering Persia and the Arabian Sea that was very advanced. He said that the inhabitants rode elephants when they went to battle. The kingdom is situated on the great Indus River. He also wrote about Anxi (Parthia), and to its west Tiaozhi (Mesopotamia). He visited Sogdiana, Kangju and Yancai (the vast Steppe). He learnt from merchants in Yuezhi that there were traders from India who brought bamboo sticks and clothes made in the western part of China known as Sichuan. Zhang Qian concluded that there were direct routes that went from mainland China to India. (Wudi sent four different groups to look for this route for trading. The route was not found. But materials recently found in the tombs of southern China verified their existence.) Zhang Qian made another trip to Wu-Sun in 119 BC. He brought with him hundreds of people, ten thousand cattle, and gold and silk as gifts to give to the heads of countries in Central Asia. Altogether, he and his envoys visited thirty-six countries.

The rise of the Kushans (who the Chinese called Yuezhi) was well-documented in the Chinese historical chronicle Hou Hanzhu. It recorded that Yuezhi envoys came to give oral teaching on the Buddhist sutras to a student in the Chinese capital around the first century BC. In fact, Yuezhi integrated Buddhism into a pantheon of many deities and became great promoters of Mahayana Buddhism. Their interactions with the Greek civilization helped the Gandharan culture and Greco-Buddhism flourish. Yuezhi is located about $3000 \mathrm{~km}$ north of India. The kings called themselves "Sons of Heaven." Yet their city layouts and palaces are quite similar to those of the Daqin (Chinese name for the Roman Empire). There are records in the Western world of the invasion in $124 \mathrm{AD}$ by Yuezhi against the Parthians, when King Artabanus I of Parthia was critically wounded and died. (Justin, Epitomes, XLII,2,2) As they settled in Bactria from around 125 BC, the Yuezhi became hellenized to some degree.

During the first century $\mathrm{AD}$, the Kushan Empire traded with the Roman Empire and also with China. They joint forces with Chinese against nomadic incursions. The Chinese general, Ban Chao, worked with them to fight against the Sogdians in $84 \mathrm{AD}$ and against the Turanians, east of the Tarim Basin in $85 \mathrm{AD}$. At that time, the $\mathrm{Ku}$ shans requested a Han princess but was denied. They then marched against Ban Chao in $86 \mathrm{AD}$ and were defeated. They paid tributes to the Chinese emperor Han $\mathrm{He}$ (89-106 AD). It is recorded in Sanguozhi (a Chinese history book of the Three Kingdoms) that in 229 AD, King of Da Yuezhi, Bodiao, sent his envoy to present tribute, and the 


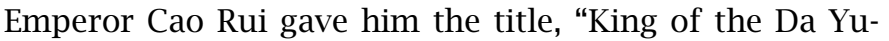
ezhi Intimate with the Wei."

During the stay of General Ban Chao in Yuezhi, he heard about Daqin (the Roman emperor) and he sent (in $89 \mathrm{AD}$ ) his deputy General Gan Ying to go to Rome. Gan Ying went through Iran and Iraq and either arrived at Antiochia, the tip of Persian Gulf, or the northeastern part of the Black Sea (Novorossiysk). Where exactly he ended up is still open to debate. Regardless, he was told by the Persians that the ocean is very dangerous, and he turned back. But the fact that the Chinese traveled that far is significant. It should be noted that Ban Chao's elder brother wrote the famous historical chronicle Hanzhu. His sister wrote a chapter of this chronicle on astronomical events. She knew mathematics well and taught the emperors and noble ladies at the court. She should had been well-read in the ancient Chinese books on mathematics. By $166 \mathrm{AD}$, the Roman merchants acting as emissaries of the Roman emperor, were able to present gifts to the Chinese emperor and teach China about the West.

Therefore, since 140 BC, the Silk Road was already very active. According to Shiji, many Chinese missions were sent. "The largest of these emissaries to foreign nations numbered several hundred persons, while even the smaller parties included over 100 members... In the course of one year anywhere from five to six to over ten parties would be sent out. "Naturally there were many engineers with knowledge of mathematics in these parties.

\section{Buddhism and the Silk Road}

The communication between China and India would have been much more active if not for the barrier of high mountains. However, the great interest in Buddhism led many monks to travel between these two ancient countries, starting around the first century. The monks back then were among the most learned men in both countries. It would not be surprising that they knew enough mathematics to communicate to each other.

Up to the Tang Dynasty, the major traffic between China and the West occurred on land through the Silk Road. The northern Silk Road went through the pastoral area which went through the Gobi Desert, through the mountain areas and finally ended up at the Black Sea, where the ancient Greeks had colonies. The second Silk Road went from Xian to Lanzhou, and through Dunhuang and then through the north of India, to Iran and to the Mediterranean Sea. See Figure 3.

In Dunhuang, there are many caves where ancient manuscripts have been found. Some of them were on mathematics, though not very sophisticated. Since the manuscripts were scattered in different places and some important ones might have been lost, it is difficult to make conclusions from those that were found. On the other hand, it is evident that Buddhist disciples played rather important roles in the Dunhuang manuscripts.

Apparently, the earliest contact with Buddhism in China may have gone back to the time of the Qin Dynasty. It was recorded that Buddhist monks from Yuezhi also came to China by $2 \mathrm{BC}$. In the year $64 \mathrm{AD}$, the Han emperor heard about the Buddah and decided to send the government official Cai Yin to India to learn Buddhism. Two high-level Indian monks came back with him and built a big temple in the capital of Luoyang.

During the period of $400 \mathrm{AD}$ and afterwards, a number of Buddhist scholars traveled between China and India: Fa Xian (399 AD) and Xuan Zang (650 AD) are the most notable ones. Among the places they traveled were monasteries such as Nalanda and Taxila which were Indian centers of scholarships, not only in religion, but also in astronomy and mathematics. Usually the monks went through the southern Silk Road to travel to India. Occasionally, they also used the Silk Road on the sea. The monks communicated with Chinese pilgrims extensively during the Sixteen Kingdoms Period (304-439 AD) and during the Tang Dynasty. The Sixteen Kingdoms were led by non-Chinese nomadic tribes, some of them are related to the Huns.

The travels of $\mathrm{Fa}$ Xian are well-documented. He walked from central China, across the Taklamakan Desert, over the Pamir Plateau, and through India to the mouth of the Hoodly, in the country of Tamluk, India. He wrote about the Kingdom of Khotan (southern arm of the Silk Road) and the Kingdom of Kashgar where the northern and southern branches of the Silk Road reunite. He went through Afghanistan (Punjab). He spent six years in India during the Gupta Dynasty. It is remarkable that besides Buddhist writings, he also wrote about the differences between Chinese and Indian calendars.

The family of the Tang emperor came from the area in the west not far from the Dunhuang area, and cultural interactions between the West and East were extensive during the Tang Dynasty. By the end of Tang period and the period right afterwards-before Sui and Song Dynasty - the "barbarian state" was too strong for the Chinese merchants to go via land on the Silk Road to trade. They started to travel via the sea. The Song Dynasty had extensive trading with countries by the Indian Ocean and all the way to the Persian Gulf and even to Africa. The last major envoy Zheng He, admiral of the Chinese fleet, was sent by the Chinese emperor seven times from 1405 to 1435, to the Indian Ocean. After that, both Ming and Qing Dynasties lost interest in interacting with the Western world in a strong manner. The direct consequence for mathematical development was very negative. Chinese mathematicians lost the broad vision and became very narrowly focused on studying the ancient developments of Chinese mathematics in its own right.

Now let us discuss the history of Indian and Chinese mathematics. We shall separate the discussion into three 
periods of time:

\section{Indian and Chinese Mathematics}

\section{The years before $500 \mathrm{AD}$}

In India, we should start with the Vedic literature: the Samhitas (1000 BC) the Brahmanas (800 BC) the Aranyakas (700 BC) and the Upanishads (600-500 BC). These are collections of hymns, prayers, sacrificial and magical formulas. Rituals and sacrifices are important in ancient India. The important ritual literature included Srautasutras, which described ways to construct sacrificial fires at different times of the year. It specifies the measurement and construction of sacrificial altars, see Figure 4. (The ritual literature are called Sulbasutras.) As was pointed out by A. Seidenberg, these sutras contain information of geometry where Pythagorean theorem can be found.

The three most important ones were recorded by Baudhayana, Apastamba and Katyayana. The one recorded by Baudhayana is the earliest, around 500 BC. This manuscript contains a general statement of the Pythagorean theorem, an approximate procedure for obtaining the square root of 2 accurate to five decimal places and squaring the circle (apparently he got the value of $\pi$ to be 3.09), and also constructing rectilinear shapes whose

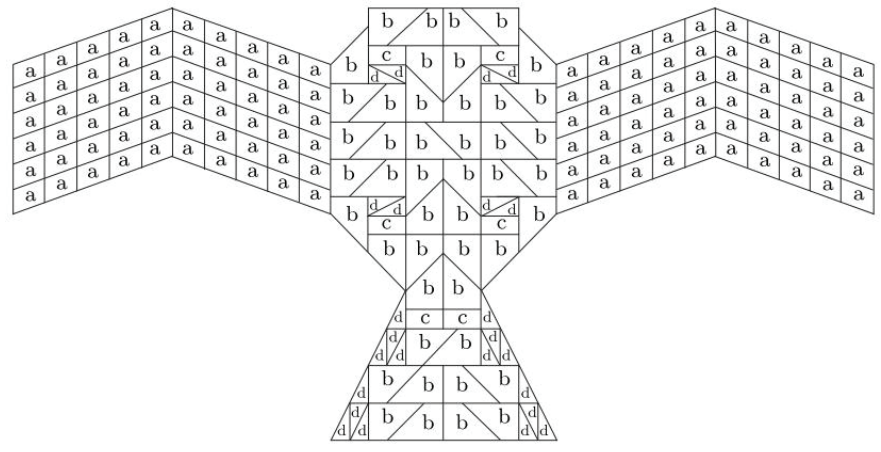

Figure 4. The first layer of a Vedic sacrificial altar in the shape of a falcon; the wings are each made from 60 bricks of type a, and the body from 46 of type b, 6 of type c, and 24 of type $\mathrm{d}$. (from G.G. Joseph, The Crest of the Peacock)

area was equal to the sum or difference of areas of other shapes. Baudhayana was followed by Apastamba and Katyayana in the next few centuries. See Figure 5 for usage of Pythagorean triples in Apastamba's Sulbasutra. Babylonian mathematicians also discussed questions on the Pythagorean theorem and their triples, the square root of two, found the value of $\pi$ to be 3.125 (as appeared in the Susa Tablet, 1600 BC). However, it is difficult to claim that the Sulbasutras derived their results from the Babylonians as there is no trace of the sexagesimal system in ancient India mathematics. As was mentioned earlier, there are also appendices to the main Vedas, where the ancient Indian paid a lot of attention to a form of writing that aimed at utmost brevity and used a poetic style to memorize literature. It may have influenced the later developments of axioms in Euclidean geometry.

The first number system of India is the Kharothi around $400 \mathrm{BC}$ to $200 \mathrm{AD}$, where the number "9" is not known to appear. The second system is called the Brahmi system which is more advanced. It appeared in the caves of Central India and is dated around $150 \mathrm{BC}$. The system eventually evolved into the Bakhshali (200-400 AD) and the Gwalior system (850 AD). They used the numbers 1 to 9 with zero. The place value principle for the number zero had to wait until it appeared in the works of the astronomer Varahamihira (587AD). This completed number system later appeared in the Arabian literature and is the current system we use today.

After the period of Vedic literature saw the rise of Buddhism and Jainism. The latter made contributions and studied mathematics for its own sake. The Jains realized different form of infinities and studied sequences. (They were interested in cosmological structures that contained innumerable concentric rings. The diameter of each ring is twice that of the previous one.)

As we have mentioned, the records of ancient China

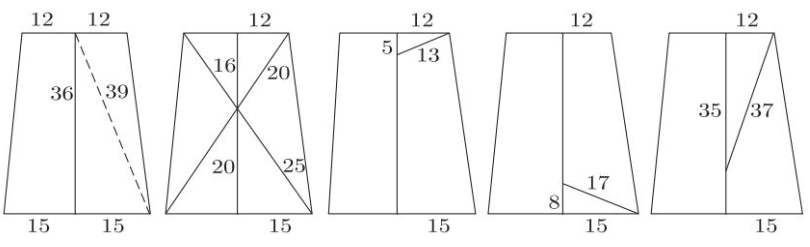

Figure 5. Trapezoid altars in the Apastamba Sulbasutra (from B. van der Waerden, Geometry and Algebra in Ancient Civilizations)

before year $1000 \mathrm{BC}$ is probably unreliable, with exceptions for those found on carvings on tortoise shells and on bronze vases. But there is no doubt that the most famous old book that is related to mathematics is the I-Ching: Book of Changes. The book is said to be written by $\mathrm{Fu} \mathrm{Xi}$ in $2850 \mathrm{BC}$. But that is most likely wrong. It is not even clear that $\mathrm{Fu} \mathrm{Xi,} \mathrm{the} \mathrm{first} \mathrm{emperor} \mathrm{of} \mathrm{China} \mathrm{ever} \mathrm{ex-}$ isted. It was quite probably written by Zhou Wen Wang around $1000 \mathrm{BC}$ and was continuously improved. At any rate, Confucius did read this book and considered it a book of great virtue. In the book, some symbols were used and the concept of yin and yang was created. And there are also sixiang (four figures) and bagua (eight trigrams). From there, it was extended to sixty-four hexagrams. The mysticism of numbers is great in the book as it has been used to this day by many to explain almost everything in our daily life. Perhaps this is similar to the Pythagorean doctrine with respect to numbers.

Magic squares also appeared in ancient China. A legend says that around 2000 BC, Emperor Yu acquired two diagrams: the first called Ho Thu (river chart) from a magical dragon horse which rose from the Yellow River and the second diagram called Lo Shu (Lo River writing) 
copied from the design on the back of a sacred turtle found in Lo, a tributary of the Yellow River. See Figure 6 . Lo Shu has the form of a three by three magic square. Ho Thu was mentioned by Confucius. But one has to be careful whether the two diagrams in Figure 6 were exactly those referred to by the I-Ching and what Confucius had in mind. Apparently, diagram (b) of Figure 6 was called the Nine Halls diagram during the period from the Qin Dynasty to the Han Dynasty. It was attributed to be the Lo Shu during the Sung Dynasty by Zhu Xi. In any case, Lo Shu is certainly the earliest recorded magic square.

The diagrams represented an important principle of Chinese philosophy: yin (female) and yang (male) in nature. The diagrams appeared in the Nine Halls of the Cosmic Temple, the Ming Tang. In 1275 AD, Yang Hui wrote in the book, Continuation of Ancient Mathematical Methods for Elucidating the Strange Properties of Numbers, that he has collected many magic squares constructed by

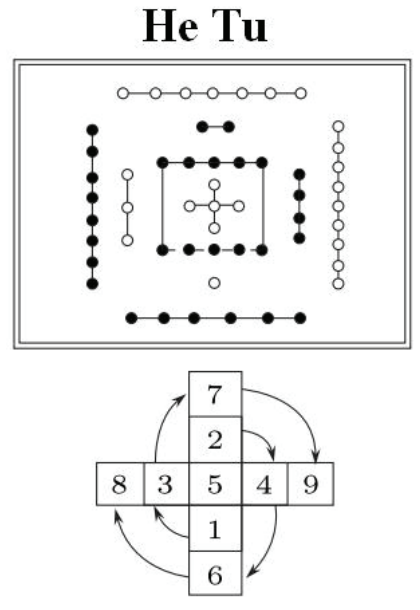

(a)

Figure 6. (a) Ho Thu and (b) Lo Shu (from G.G. Joseph, The Crest of the Peacock).

(b)

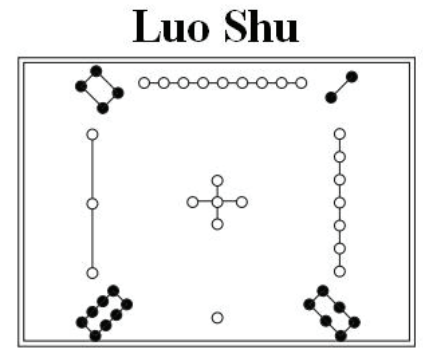

\begin{tabular}{|l|l|l|}
\hline 4 & 9 & 2 \\
\hline 3 & 5 & 7 \\
\hline 8 & 1 & 6 \\
\hline
\end{tabular}

the ancient Chinese. He also gave properties of the squares and classified the squares up to order 10. In 1880, Pao Chi Shou constructed magic cubes, spheres and tetrahedrons. Even now, some Chinese scholars are still fascinated in numerology and number mysticism related to I-Ching.

I-Ching is deeply influential in the intellectual circles through the teaching of Confucius. Its system of base 60 is used by many Chinese scholars even today. However, for more practical issues such as trading or bookkeeping, the decimal system was used in a large scale by $250 \mathrm{BC}$ and perhaps even much earlier. (It had been found on the tortoise shells that dates back to $1200 \mathrm{BC}$.) In fact, some arithmetic instrument were used. (Archeologist had dug up bamboo or ivory sticks that were used for arithmetics as early as the Qin Dynasty, around 220 BC. See Figure 7. The ancient Chinese decimal system, see Figures 8 and 9, is interesting in that it operated without the "zero" number but yet it was still very effective and not ambiguous. This is in contrast to the number system without zero that created ambiguity in the Babylonian literature. The invention of "zero" by the Indian around $700 \mathrm{AD}$ is a tremendous contribution to mathematics. On the other hand, the Chinese bamboo sticks can be considered as the world's oldest simple computing instrument.

After the I-Ching, there was Chou Pei Suan Ching. This book's written date has been much debated for a long time. It contains a conversation of Duke Zhou Gong and his minister Shang Kao around 1000 BC. (The conversation most likely never occurred. It may be an excuse for the author to give some importance for the mathematics in the book.) The Pythagorean triple $(3,4,5)$ was mentioned in this book. This book was supposed to consist of government documents on how to use mathematics to understand astronomical events. It was collected together to form a book around $235 \mathrm{BC}$ to $156 \mathrm{BC}$. The editor was quite likely to be Zhang Chang around 165 BC. Between 190-220 AD, a famous mathematician called Zhao Shuang made detailed commentary on Chou Pei and added in some original ideas, including the proof of the Pythagorean theorem as seen in Figure 10. (The general statement of the Pythagorean theorem was known much earlier as was described in Chou Pei.)

The most important classic of Chinese mathematics is the Nine Chapters on the Mathematical Art. This book also consists of collections of government documents on mathematics related to events on earth. Nobody can be sure how old this book is. But it is fair to say that the book was also edited by Zhang Chang around 200 BC. Being a high-level government officer, it is likely that he made extensive use of ancient government documents, all the

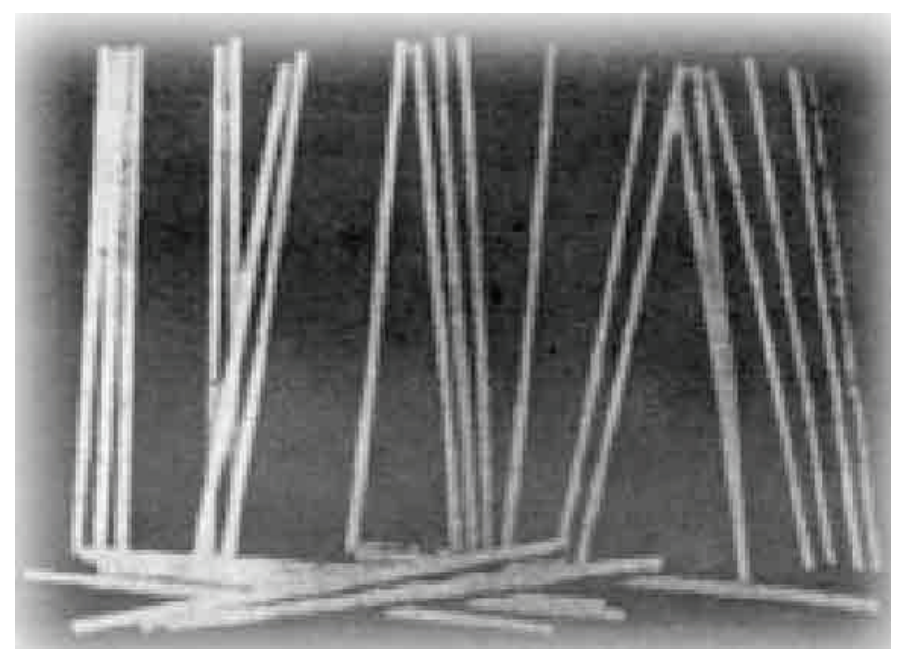

Figure 7. Ancient Chinese ivory calculating sticks (from Shanghai-Bavaria virtual campus telecenter)

way back to Zhou Gong, about 1000 BC. In this book, there are questions asked on various topics in mathematics: calculations of volume, area, equations; approximations of square root and cubic root; and the Pythagorean theorem and its triples. The Euclidean algorithm was used 
extensively and the Gaussian elimination for matrices was introduced. (The Chinese calculations using bamboo sticks led naturally to matrices.) Answers to the questions raised in the book could had led to generalizations. But no general statements were stated.

Around 1984, a reasonably complete manuscript called the Book of Mathematical Art written on bamboo sticks (about 190 sticks altogether) was excavated from an old tomb of the Han Dynasty in Hubei Province. The tomb was buried around $186 \mathrm{BC}$ and therefore the book should had been completed before this date. There are many overlaps in the contents between this book and the Nine Chapters, though it is not as advanced. One may speculate that the editor of the Nine Chapters collected many ideas from these old manuscripts.

Several spectacular works can be found in the third century AD after the Nine Chapters. Liu Hui (208-263 AD) was the one who wrote a great detailed commentary on the Nine Chapters. His commentary exhibited a great deal of his own works. A rigorous proof of the Pythagorean theorem in the Euclidean manner was given. (Liu Hui did not actually draw pictures of his proof. Hence, modern understandings were needed to interpret this classical Chinese writing.) He computed $\pi$ up to four decimals

\section{$\begin{array}{llllllllll}\text { Vertical : } & \mid & \| & || & \|\| & \|\| \| & \top & \pi & \pi & \pi||\end{array}$ Horizontal : $\quad-\quad=\equiv \equiv \perp \equiv \equiv$$$
\begin{array}{lllllllll}
1 & 2 & 3 & 4 & 5 & 6 & 7 & 8 & 9
\end{array}
$$ \\ Figure 8. Thirteen number inscriptions found on Chinese oracle bones (from Shanghai-Bavaria virtual campus telecenter)}

using a 192 sides regular polygon approximation of the circle. He discovered the principle that the volumes of two solids are the same if the cross sections with the same height has equal area. Extensive discussions of sub-dividing a solid into smaller and smaller prisms can be found. He also developed further the concept of a limit and gave geometric interpretations of finding the cubic root of a number. His computation of $\pi$ was followed in $480 \mathrm{AD}$ by a computation of Su Chung Chih who computed $\pi$ up to 7 digits using 24576 polygons.

The need of refining the calender drove the Chinese to look into equations of undetermined type (of first degree). It was first written about by Sun Tze, in Sun Tze Suan Ching, where the concept of congruence was introduced and the Chinese remainder theorem was discovered. According to the investigations of Qian Bao Zong, this book was written around $400 \mathrm{AD}$, although there are suggestions that it was written around $300 \mathrm{AD}$.

The research was generalized to a system of linear equations of undetermined type in the Song Dynasty. The work of Sun Tze depended on the understanding of the Euclidean algorithm which was written in the Nine Chapters, and most likely went back to the ancient days of Chinese arithmetic.
Su Chung Chih (429-500 AD) and his son are two remarkable mathematicians of this period. He devoted a great deal of his time to astronomical calculations. Besides the above-mentioned precise calculations of $\pi$, he was able to solve any complicated system of undetermined linear equations of 12 variables.

\section{The period between $500 \mathrm{AD}$ and $1450 \mathrm{AD}$}

There was a manuscript dug up in Bakhshali in 1881. It was written in the old form of Sanskrit. The date of this manuscript is still undetermined, presumably from around $500 \mathrm{AD}$, but it is the earliest evidence that Indian mathematics started to be free of any religious and metaphysical associations. It has many resemblance with the Chinese Nine Chapters, including the style of writing. But the content is not as advanced as the Nine Chapters.
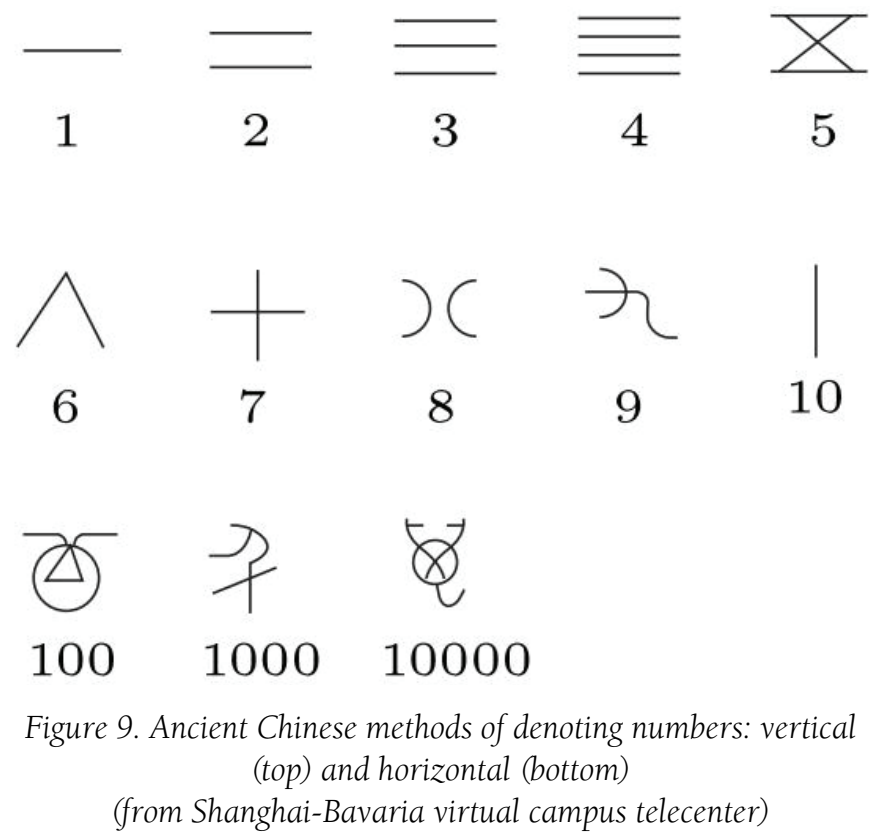

The first major mathematician-astronomer of India was Aryabhata I, who wrote the book Aryabhatiya. The work was around $500 \mathrm{AD}$. The work was done in Kusumapura, the imperial capital of the Gupta Empire. It was recorded by Umasvati (in $200 \mathrm{AD}$ ) that this is the place where a famous school of mathematics and astronomy was formed. The Aryabhatiya is concise and is essentially a systemization of the results contained in the older Siddhantas. The mathematics section of this book contains only 33 verses. Here we find the rules for arithmetic operations, simple and quadratic equations, and indeterminate equations of the first degree. Sine and cosine are introduced here. He also computed $\pi$ to be 3.1416. While trigonometry owed its foundation to Hipparchus (150 BC), Menelaus (100 AD) and Ptolemy (150 AD), Indian trigonometry began to take shape in the modern form during this period (partially due to the interest of astronomy). Important identities of the trigonometric func- 
tions were found by Aryabhata I, Varahamihira, Brahmagupta and Bhaskaracharya. Especially central to this development was Bhaskaracharya, who also studied indeterminate equations of second degree: Pell's equation. Based on the work of the astronomer Manjula (930 AD), Bhaskaracharya derived the differential of the sine function.

In the south, Kerala was the place where early form of calculus was developed. In $850 \mathrm{AD}$, Govindaswami produced a rule for second order interpolation to compute intermediate functional values. A particular case of the Newton's interpolation formula was known to them. Taylor series up to the second order term was also known to them. The important mathematician in this area was

\section{Chordal Graph}

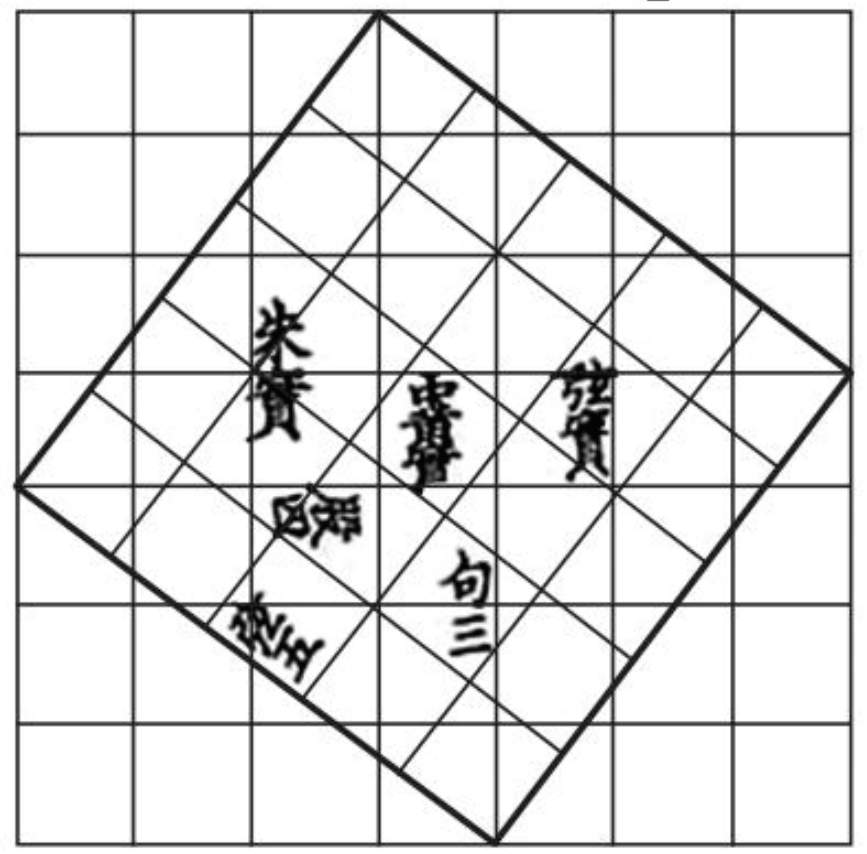

Figure 10. The proof of the Pythagoras Theorem in Chou Pei Suan Ching

(from B. van der Waerden, Geometry and Algebra in Ancient Civilizations)

Madhava (1400 AD) who discovered power series for $\pi$, for sine, cosine and arc tangent functions. (Though the original sources have not been found, the discoveries were referred to by later writers.)

It appears that Indian mathematicians started the subject of mathematical analysis long before Newton and other Western workers. While the knowledge of differential calculus is impressive and perhaps had influenced Western works a few hundred years later, it is not as complete. Integral and differential calculus were not combined as well as by later workers. But nevertheless, the Indian advances during this period are truly impressive.

In the Sui Dynasty of China, there were records of
Indian astronomical and mathematical texts, such as the Brahmam Suan Fa (the Brahman arithmetical rules) and Brahman Suan Ching (Brahman arithmetical classic).

The records of the Tang Dynasty contain the names of Indian astronomers. One of the Indians, whose Chinese name was Chu Tan Hsia Ta (Gautama Siddharta), was reputed to have constructed a calender, based on the Indian Siddhantas, by order of the first emperor of the Tang Dynasty (in $718 \mathrm{AD}$ ) . The text contains Indian numerals and operations and sine tables. Yabbuchi (1954) located a surviving block-print text which contains Indian numerals, including the use of a dot to indicate zero. There is also a sine table at intervals of 3 degree 45 arc for a radius of 3438 units, which are the values given in the Indian astronomical texts Aryabhatiya and Surya Siddhanta.

In both the Sui and the Tang Dynasties, the government appointed scholars to study mathematics, and for most part, mathematics was part of the civil service examinations given by the government. There were ten books on mathematics that were tested on. The rank of the Officer in Mathematics was the lowest in the government. Of these mathematicians, Wang Xiao Tong (around $620 \mathrm{AD}$ ) was the most famous one. He transformed the problem of geometry into algebra. He was the first to study cubic equations which he claimed to be able to solve.

As was mentioned earlier, Admiral Zheng He brought more than twenty thousand people with him to travel to the Indian Ocean seven times during the period of 1405 to 1433. His envoy certainly went to Kerala, in southwest India, where Madhava and other Indian mathematicians were developing early forms of differential calculus. One naturally wonder whether there were any mathematical exchanges between the Chinese and Indians then?

It is a curiosity that despite the official examinations on mathematics, the Tang Dynasty did not produce as many good mathematicians as we would expect. I believe that the Chinese system of examinations discouraged innovative thinking and that the low rank of the officers in charge of the mathematics examinations could hardly measure up to the aspirations of young scholars of that period.

Building on the Nine Chapters, the Song Dynasty produced quite a few outstanding mathematicians. Chin Chiu Shao (1250) numerically solved equations of high order based on the use of what we call Pascal's triangle method for the extraction of roots. The discussion of the Pascal triangle was treated by Yang Hui and Chu Shih Chieh earlier. Yang Hui reported that this work was due to Chia Hsien in 1050. Chin Chiu Shao also extended the Chinese remainder theorem to more variables. Another mathematician named Kou Shou Chih (1275) derived the cubic interpolation formula which now goes by the name of Newton-Sterling formula.

The above mentioned method of solving high degree equations is now called Horner's method. It dates back to the Nine Chapters. The method of extracting cubic root 
appeared in the Arabian mathematician al-Nasawi (1025). Later al-Kashi (1450) extracted roots of higher degree.

There were many good mathematicians producing important works in this period. I regret that some of them may not be mentioned here.

\section{The period between 1450 and 1900}

It is still a mystery that both Indian and Chinese mathematics did not develop much during this period of time. Any progress made certainly was nowhere comparable to that made in the Western world during this period.

During this time, Europeans enjoyed tremendous economic growth and academic freedom compared with the Asians. Their pursuit of truth and beauty for its own sake, was not something that the Asians can compare to. A lot more study is needed to uncover the reason behind our decline during this period.

The mathematical developments after the Song Dynasty declined. By 1582, the Italian Jesuit Ricci came to China and translated the first six books of Euclid's Elements. A Chinese mathematician by the name Xu Guangqi helped Ricci with the translation. Xu expressed his amazement on the logical structure appearing in Euclid's book. It was unfortunate that Euclid's powerful deductive method was never developed in China until the last century. (In fact, the translation of the last chapters of Euclid's Elements was only completed in the 19th century by a Chinese mathematician called Li Shanlan.) The Jesuits may have brought back to Europe some of the mathematics of China. It may be interesting to note that the arrival of Ricci occurred roughly 100 years before the publishing of Newton's Principia in 1687. By then, the logical structure and the deductive power of Euclid 's methods already had a deep influence in the Western world. Not only did they help clarify many puzzles, they also opened up new horizons in mathematics and physics. Such influence did not even begin to take root in Asia until almost two centuries later.

The Qing Dynasty mathematicians are not as original as those of the Han or Song Dynasty. And despite the efforts of the great emperor Kangxi of the Qing Dynasty, who was extremely interested in mathematics and hired several missionaries in his court, the glory of Chinese mathematics did not continue. Perhaps this exemplified the academic freedom in both the Han and the Song Dynasties. Besides that, the examination system during the Qing Dynasty did not pay enough attention to mathematics, (while that of Song and Tang placed more emphasis). The overemphasis of practical use of mathematics finally showed its weakness. The overwhelming success of mathematical development in Western world which combined the power of both abstract and practical application is so great that it took a long time for the Asians to catch up.

Among the three most famous ancient problems in mathematics were:

1. the parallel postulate

2. squaring the circle

3. the trisection of an angle

The Chinese and Indian mathematics only touched the second one. The first one led to the introduction of hyperbolic geometry while the second one is related to Galois theory. Both of them have had a great impact on modern mathematics.

\section{0 till today}

Both Indians and Chinese mathematics started to recover.

In modern India, the most original mathematician was no doubt Ramanujan, who changed our views on modular forms. His shear mathematical power was tremendous and many of his ideas still need to be further explored even today. Ramanujan visited England and learned a lot from Hardy, who was able to explain some of Ramanujan's ideas. Ramanujan made decisive contributions to number theory at that time. The tradition of number theory was carried on by S.S. Pillai on the Waring's problem, by S. Chowla and by K. Chandrasekharan.

There are many Indians who left India to learn from the Western world. The great mathematician Harish-Chandra who went to England to study with Dirac, developed on his own the foundations for the theory of representation of noncompact groups. It is difficult to measure his deep influence in analysis and number theory. There are also many other Indian mathematicians who played very important roles in modern mathematics. The works of S. Minakshisundaram and Pleijel (Canadian) on eigenvalues of Laplacian through the heat kernel expansion is spectacular: a subject that Herman Weyl thought highly of. When Patodi further developed this subject, it finally led to a new analytic local form of the Atiyah-Singer index theorem which has had a tremendous influence in modern works. The contributions made by Indian mathematicians Raghunathan, Narasimhan and Seshadri on discrete groups and on vector bundles over algebraic curve were also fundamental.

Many outstanding Indian mathematicians have stayed in the West and became leaders in their respective fields. This list includes Abyanhker (algebraic geometry), Chandrasekharan (number theory), Kulkarni (geometry), R. Narasimhan (several complex variable), Nori (algebraic geometry), Pandharipande (algebraic geometry), Parimala (algebra), Prasad (discrete group theory), Varadarajan (representation theory) and Varadhan (probability and analysis). Chandrasekharan had great influence in the International Mathematics Union where he was the president for one term and secretary for two terms. Varadhan was awarded the prestigious Abel Prize. We are also seeing the growth of the second generation of mathematicians of Indian ancestry (born in the West). The 
works of young mathematicians such as Manjul Bhargava and Chandrashekhar Khare, both on number theory, Ravi Vakil on Gromov-Witten invariants and that of (IIT Kanpur's) Manindra Agrawal, Neeraj Kayal and Nitin Saxena on an algorithm for testing prime numbers have received much international recognition recently.

The founders of Indian statistics are P.C. Mahalanobis and P.V. Sukhatme. The latter got his Ph.D. from the University of London in 1936. He settled in Delhi and formed a group of statisticians in the Indian Council for Agricultural Research. Mahalanobis founded the Statistical Institute in 1931 and also Sankha, the India Journal of Statistics in 1933. By the late 1950s, the Indian Statistical Institute became a center of national importance. Their associates such as R.C. Bose, C.R. Rao and S.N. Roy became prominent statisticians and are world famous. Nowadays, statistics plays an influential role in Indian society and government: there are the Ministry of Statistics and Programme Implementation, the Indian Statistical Service, the National Sample Survey Organization, the Indian Agricultural Statistics Research Institute, the Institute for Research in Medical Statistics, etc.

In the last hundred year of Chinese history, we saw the rise of several stars in mathematics: Shiing-Shen Chern, Loo-Keng Hua and Wei-Liang Chow. Chern obtained his bachelor's degree studying under Lifu Jiang at Nankai University and his master's under Guangyuan Sun at Tsinghua Univestiy. Hua was self-educated and was discovered by King-Lai Hiong and Wuzhi Yang, the leading professors at Tsinghua University. (Yang was the first Chinese mathematician to earn a $\mathrm{PhD}$ in number theory, from Chicago.) Hua was offered to work in the library as he had no high school degree. Both Chern and Hua were in Tsinghua at the same time around 1930. Besides the influences of Hiong, Sun and Yang at Tsinghua, there were important visits to China by some great professors from Europe and America in the 1930s. They included Norbert Wiener, Osgood, Blaschke and Hadamard. Chern studied with Blaschke in Hamburg and later went to Paris to study with Cartan. It may be interesting to note that Chern was offered a job at the Tata Institute in 1950 which he declined. Hua went to Cambridge to study with Hardy and later learned a lot from the Russian number theorist Vinogradov. Chow also went to Germany in 1932 and studied under van der Waerden. He returned to China to teach in Central University, and then became a businessman before leaving for Princeton in 1947 to study with Lefschetz. His great works were done in Princeton. These three great mathematicians were in China during the war with Japan.

A great number of brilliant students were trained by them. Chern helped his teacher run the Mathematics Institute of the Academia Sinica until 1949, when he left for America. During this period, he trained many first class students on topics related to geometry and modern topology. He had just finished his fundamental work on Chern classes and Gauss-Bonnet theorem in Princeton
(1944). Hua was sent by the government to America to learn the secrets of the atomic bomb, which he never did. But when he returned to China in 1950, he did initiate several important branches of mathematics. Most notable were several complex variables and analytic number theory, where he trained a group of students on the theory of classical domains, the Waring's problem, and the Goldbach problem. In the latter problem, the Chinese gained the world's attention when Jingrun Chen proved that every sufficiently large even number can be written as either the sum of two primes or a prime and the product of two primes. Chow left China right after the war. His great insights into algebraic geometry have influenced the subject deeply. Unfortunately he did not train many students. There were other significant works in complex analysis and in Fourier analysis. But none of the achievements compared with the above mentioned figures.

On the other hand, the Chinese are very strong in the developments of applied mathematics. The leading figures are Chia-Chiao Lin, Yuan-Cheng Fung, Theodore Yao-Tsu Wu, Kang Feng and Pao-Lu Hsu. Lin did pioneering works on the density dynamics of galaxies. Fung pioneered bio-fluid dynamics and $\mathrm{Wu}$ contributed in a fundamental way to ocean fluids. Kang Feng is one of the founders of the finite element method which formed the foundation of numerical computations. And Pao-Lu Hsu is one of the founders of modern probability and statistics. He made fundamental contributions to the subject of probability and statistics such as partially balanced incomplete block designs and the asymptotic theory of empirical distributions. He is the role model for many outstanding Chinese statisticians that settled in America. Perhaps his return to China in 1947 has inspired some of these statisticians to contribute to Chinese society.

In the past century, Chinese mathematics had gone through three different stages of developments. The first is around the time of late 1920s to 1940s-when the first group of Ph.D. students came back home to teach, great mathematicians came to visit China, and new textbooks from Europe and England were used. When Chern and Hua came back in the mid-1930s, geometry, algebra and number theory started to take roots. There were also developments of complex analysis by King-Lai Hiong, who studied in France, Fourier analysis by Jiangong Chen who was educated in Japan, differential geometry by Buqing $\mathrm{Su}$ who was trained in Japan and algebra by Chiungtze C. Tsen who was trained in Germany. During this period, many young people were trained by this group of leaders. Among them were Kuo-Tsai Chen, Kai Lai Chung, Ky Fan, Chuan-Chih Hsiung, Zhao Ke, Hsien-Chung Wang, Zhikun Wang, Wen-Tsun Wu, Zhida Yan and Chung-Tao Yang. Besides Ke, Wang, Wu and Yan, the rest left China. Hsiung is the founder of the Journal of Differential Geometry.

The second period was the time when Hua went back to China in 1950 to head its mathematical development. Many young mathematicians were trained by him, and 
also by Chen, Hiong and Su. While this group of mathematicians focused on pure mathematics, the prominent numerical analyst, Kang Feng, initiated the subject of computations in China. The famous statistician, Pao-Lu Hsu, who had returned in the forties also led China to the forefront of statistics research. Some of the other most notable people of this period are Jingrun Chen, Xiaxi Ding, Sheng Gong, Boling Guo, Chaohao Gu, Jiaxing Hong, Hesheng Hu, Shantao Liao, Qikeng Lu, Chendong Pan, Zhong-Ci Shi, Zhexian Wan, Guangyin Wang, Yuan Wang, Daoxing Xia, Lo Yang, Guanghou Zhang, Jiaqing Zhong and Yulin Zhou. This group of younger generation mathematicians did very good works in the early sixties and thereafter. Somewhat later, many good Chinese mathematicians were produced in Hong Kong and Taiwan, such as Ching-Li Chai, Tony Chan, Gerald Chang, Sun-Yung Alice Chang, Mei-Chu Chang, Shiu-Yuen Cheng, I-Liang Chern, Fan Chung Graham, Wu-Chung Hsiang, Ming-chang Kang, Tze Leung Lai, Tsit-Yuen Lam, Naichung Conan Leung, Peter Li, Wen-Ching Winnie Li, Bong Lian, Chang-Shou Lin, Song-Sun Lin, Tai-Ping Liu, Chiang C. Mei, Ngaiming Mok, Tzuong-Tsieng Moh, Wei-Ming Ni, Yum-Tong Siu, Yung-sheng Tai, Luen-Fai Tam, Chuu-Lian Terng, Wing Hung Wong, Chien-Fu Jeff Wu, Hung-Hsi Wu, Andrew Chi-Chih Yao, Horng-Tzer Yau, Stephen Shing-Toung Yau, Lai-Sang Young and Jin Yu. Most of them were trained in graduate schools in America and many stayed in the US.

The third period was the time after the Cultural Revolution till today. Many young students were trained in American graduate schools and many of them have remained in the US. A good sample can be found in those who received prestigious awards: Jin-Yi Cai, Raymond Chan, Chiun-Chuan Chen, Chong-Qing Cheng, Ding-Zhu Du, Weinan E, Jianqing Fan, Pengfei Guan, Lei Guo, Thomas Yizhao Hou, Lizhen Ji, Shi Jin, Jun Li, Fanghua Lin, Xihong Lin, Ai-Ko Liu, Chiu-Chu Melissa Liu, Jun Liu, Kefeng Liu, Feng Luo, Xiaoli Meng, Sheng-Li Tan, Gang Tian, Daqing Wan, Chin-Lung Wang, Mu-Tao Wang, Shicheng Wang, Xu-Jia Wang, Sijue Wu, Nanhua Xi, Jie Xiao, Zhou-Ping Xin, Zhiliang Ying, Guoliang Yu, Jiu-Kang Yu, Shou-Wu Zhang, Xiangyu Zhou, Xin Zhou, Xiaohua Zhu and Xiping Zhu.

The economic situation of China has improved tremendously in the past twenty years. It is expected that many brilliant Chinese mathematicians will go back in the future. This shall make a tremendous difference for the future of Chinese mathematics. As a notable example, about three years ago, Andrew Chi-Chih Yao, the first Chinese recipient of the Turing Award, resigned his job from Princeton University and settled down at Tsinghua University. This is significant as it demonstrates the desire of overseas Chinese to help and participate in the development of mathematics in China.

Overall, the overemphasis of Chinese mathematics on practical applications in its history has been an obstacle for the advancement for core mathematics, which in turn is the major obstacle for the advancement of applied mathematics. If we look at the past history of the development of mathematics in China, it is amazing that the Chinese placed so much emphases on the development of their forefathers as compared with creating innovative ideas for the new frontier of science. They are much less adventurous compared with our Western colleagues. A good example is the outstanding mathematician, Liu Hui, and later mathematicians of the Song Dynasty. They spent most of their time understanding the ancient book of the Nine Chapters. They made a great deal of advances in mathematics that way. But it is different from developing new ideas to understand the beauty of nature and creating new subjects. On the other hand, Sun Tzu's development of the Chinese Remainder Theorem is original and spectacular.

\section{Concluding Remarks}

Historical events have shown that when Indian and Chinese societies are open and interacting with foreign countries, outstanding mathematical developments took place. When the countries closed their borders or looked inward, the mathematical developments were quite different and often with no significant progress. A closed society not only loses the stimulation of fresh new ideas from the outside, but its scholars also tend to lose their objectivity in their judgements. Clearly cultural and economic exchanges are very important for mathematical advancement.

For the last couple of years, both India and China have been experiencing double-digit economic growth. Unless some unforeseen events arise, large-scale conflicts with other countries seem unlikely (and are of course, highly undesirable by all people in the region.) In a time of peace and with a reasonable accumulation of wealth, the governments of both countries shall continue to invest in mathematics. Highly talented mathematicians in India and in China will no doubt work toward making important contributions for the good of mankind. Many prominent mathematicians will also come back and settle down in both countries. We have already seen many of our friends from other countries spending their precious time to visit us and deliver important lectures. In a short period of time, we will see our subject blossom again in our fertile land.

The great achievements of Ramanujan and Harish-Chandra have inspired generations of Indian mathematicians to work on both number theory and representation theory. The visits of Weil, Borel, Siegel, Samuel, Eilenberg, Mumford and Deligne have encouraged development of number theory, discrete group theory and algebraic geometry in India. Chern was an important role model and his works inspired much work in geometry and topology in China. Hua's work on analytic number theory and several complex variables have now established a "tradition" in Chinese mathematics. Chow's work is fun- 
damental in algebraic geometry, but the impact of his work in China is not as clear as compared with that Chern and Hua, perhaps because Chow resided in America for most of his active years.

I am sure that a close collaboration between Indian and Chinese mathematicians will lead to great advancements in modern mathematics. Without regards to national boundaries, we can share our role models and communicate with each other, treating students from the other country like one's own.

In ancient days, the pursuit of truth and beauty was perhaps more focused on issues relating to those of agriculture, building constructions, ritual worship, music and astronomy. We expect that the globalization of economies will also promote the unification of different areas of mathematics. In fact, the unification of mathematics is a trend of modern mathematics. In the past century, great achievements in mathematics often spanned different disciplines of mathematics, physics and engineering. These include the works of Cartan, Hodge, Weyl, Lefschetz, Weil, Chern, Morse, Deligne, Hirzebruch, Atiyah, Singer, Langlands, Wiles, and more recently, the proof of the Poincaré conjecture which used geometric analysis, a subject that is not so familiar for classical topologists.

There are an abundance of mathematical developments that are influenced by the desire for a unifying theory in fundamental physics. String theory has inspired many different structures in mathematics to be merged together as one in a natural manner. This whole subject is rather complicated as it involves many different branches of mathematics and physics. I believe the unification of different disciplines of mathematics will occur in the future and that this will require the efforts of many, many mathematicians. Besides interactions with fundamental theories of physics, questions with practical applications have also inspired important developments in mathematics. This includes fluid dynamics, numerical calculations, theory of complexity, graph theory, etc. I am sure our youth in India and China will contribute to this great endeavor and discover the beauty that we all love, that is mathematics.

\section{Appendix. A Letter To Yau}

\section{From David Mumford, Brown University}

Dear Yau,

Thank you for asking me to add some comments to your very interesting survey of Indian and Chinese mathematics. Like you, I am an amateur in the History of Math but have pursued this study with much interest recently. First, a plea: it is very hard for Westerners not speaking Chinese to keep straight the Chinese names of people, places, etc., due to the shift from the older Wade-Giles system of transliteration to the newer officially sanctioned Pinyin transliteration.
Thus I know well the Zhou Bi Suan Jing (as it is called in pinyin) and was confused when you called it the Chou Pei Suan Ching (following Wade-Giles)!

i) Concerning your survey of the earliest roots of math in India and China, I'd like to add my understanding of what came first and why. I believe there are three problems which were of great importance to the earliest civilizations and which required math. The earliest which is clearly documented is the need to track transactions of goods. This is found c. 3000 BCE in Mesopotamian artifacts and seems to have led directly to their place value sexagesimal arithmetic. Another found on tablets from the late 3rd millennium BCE is the need calculate the areas of fields for taxes and inheritance. It is this, plus the desire to lay out buildings and towns in rectangles that seems to be the main impetus for the discovery of "Pythagoras's" theorem. Babylonian tablets, c.1800 $B C E$, even show the three-dimensional version of this theorem used to calculate the distance from the inner, lower, left corner of a gateway to the outer, upper, right corner. And finally predicting the motions of the sun, moon, planets and stars is clearly a third source of mathematical problems?-for instance the desire to find the least common multiple of all the periodic effects.

ii) In tracking the earliest roots of Indian math, a most important fact to understand is their ancient oral tradition in which whole texts are memorized and passed down from father to son and to pupil. This oral tradition goes back at least to the late 2nd millennium $B C E$ when the Rg Veda was composed. They had memorization tricks for ensuring that no syllable was left out, guaranteeing that the composition was passed on without error. There were even several competing ways to speak large numbers and even tables of numbers to make them memorable, using either nonsense syllables or metaphors for each digit. Written texts only came in a millennium later and even today have not completely displaced the oral tradition in conservative Brahmin families.

iii) As you describe, Alexander, coming from the West and the Yuezhl/Kushan, coming from the East, both reached India and thus linked up in one big chain on which some math might have been transmitted. Ptolemy's book "Geography" is a remarkable source illustrating the later Greek knowledge of the Silk Road and their estimate of the longitude of China. Taxila was a key center of learning where Indo-Greeks and Chinese monks met. But we really don't know whether any Greek math books made it to there. Greek astronomy certainly did (e.g. the Indians knew the radius of the earth essentially correctly and divided the ecliptic into twelve parts following the Greeks). Moreover, I don't know of any evidence that the Yuezhi acted as a conduit for mathematical or astronomical 
ideas between India and China. Even later in the Tang, the Indian astronomers who came to Chang'an seem to have had only minor influence on subsequent Chinese calendars.

iv) Panini seems to me and many others a seminal figure in drawing Indian mathematicians in the direction of algorithms and especially recursion as a technique. Perhaps the emphasis on algorithms pushes any felt need for proofs into the background. In fact, Indian philosophers rejected the law of the excluded middle. In emphasizing algorithms, Indian math closely parallels Chinese math.

v) It is strange to me that the idea that "India invented zero" is nearly universally accepted. Place value, i.e. writing numbers as sequences to some base, goes back before 2000 BCE in Mesopotamia, is used orally in the Vedas and used from ancient times on Chinese counting boards. The idea of zero merely amounts to replacing gaps by an explicit symbol or marker and this is done in some later Babylonian tablets (Seleucid period), cf. Neugebauer's book "The Exact Sciences in Antiquity".

vi) You omit some of my heroes in Chinese math! I love $Z u$ Xuan's calculation of the volume of the sphere $c$. 500 CE and Li Zhi/Zhu Shijie's invention of elimination theory for polynomials in more than one variable, the equivalent of resultants c.1300 CE. I also love Aryabhata's discovery of the finite difference form of the differential equation for sine, c. 500 CE. Their approach to calculus was similar to Leibniz's, starting with finite differences and finite summations. They studied summing powers of integers and this led them for instance to the fact $\int x^{k}=x^{-k+1} /(k+1)$.

Best regards, David

\section{References}

[1] B. Datta and A. N. Singh, History of Hindu Mathematics: a Source Book, Asia Publishing House, New York 1962.

[2] G. G. Joseph, The Crest of the Peacock: non-European Roots of Mathematics, Penguin Books, New York 1991.

[3] Di Li, Chinese Mathematical History: from the Early Ancient Period through Five Dynasties, Jiangsu Education Publishing House, 1997.

[4] I. Marx, Travels of Hsuan-Tsang-Buddhist Pilgrim of the Seventh Century, Silkroad Foundation, 1997.

[5] D. E. Smith, History of Mathematics, Dover Publications, New York 1958.

[6] B. L. van der Waerden, Geometry and Algebra in Ancient Civilizations, Springer-Verlag, New York 1983.

[7] B. L. van der Waerden, A History of Algebra: from al-Khwarizmi to Emmy Noether, Springer-Verlag,

New York 1985. 